\title{
IMPORTÂNCIA DA PSICOLOGIA DO ESPORTE PARA TREINADORES
}

Danilo Reis Coimbra

Simone Salvador Gomes

Felipe Carvalho

Renato Ferreira

Felix Guillen

Renato Miranda

Mauricio Bara Filho

\section{Resumo}

O objetivo do estudo foi verificar a importância da psicologia do esporte para treinadores. A pesquisa foi realizada com 59 treinadores (idade 34,98 $\pm 10,26$ e tempo de prática 9,67 $\pm 8,91$ ) de modalidades coletivas e individuais que responderam a um questionário especifico sobre a psicologia do esporte na prática esportiva. Os treinadores consideram todos os aspectos do treinamento importante, porém poucos conhecem o aspecto psicológico. A grande maioria considera importante a presença do psicólogo na equipe, mas deve atuar auxiliando o técnico, porém poucos concordam que ele possui conhecimento sobre o esporte. Futuros estudos são necessários para apontar quais são os temas da psicologia do esporte que os treinadores necessitam em sua pratica.

\section{Palavras-Chave}

Importância; Psicologia do Esporte; Treinadores.

\section{THE IMPORTANCE OF SPORTS PSYCHOLOGY FOR COACHES}

Danilo Reis Coimbra

Simone Salvador Gomes

Felipe Carvalho

Renato Ferreira

Felix Guillen

Renato Miranda

Mauricio Bara Filho

\begin{abstract}
The purpose of the study was to verify the importance of the sport psychology for coaches. The research accomplished with 59 coaches (age 34.98 \pm 10.26 and practice time $9.67 \pm 8.91$ ) to several sports and different levels that answered a specify questionnaire about sport psychology in sports practice. The coaches consider all training aspects important; but few of them have knowledge about the psychological aspect. The great majority considers the psychologist presence important for the team, aiding, however few agree that he has knowledge about sport. Futures studies are needed to appear which are the themes of the sport psychology that the trainers require in their practice.
\end{abstract}

\section{Key-Words}

Importance, Sport psychology; Coaches 


\section{INTRODUÇÃO}

O esporte tem sido considerado um dos maiores fenômenos sociais do século, pois está presente no cotidiano de todos, seja como praticantes ou espectadores. Diversas são as modalidades esportivas que vêm ganhando cada vez mais atenção principalmente da mídia e de empresas interessadas em relacionar sua marca a atletas vitoriosos. Conseqüentemente, o esporte de rendimento vem se tornando cada vez mais organizado e profissionalizado, fazendo com que atletas apresentem sempre a sua máxima performance, pois exige-se que ele ultrapasse limites a cada competição (VIADÉ, 2003; BARA FILHO; MIRANDA, 1998; SAMULSKI, 2002; SOUZA FILHO, 2000; BECKER JÚNIOR, 2000; FONSECA, 2001).

Desta forma, o esporte contemporâneo tem agregado em torno de si um número cada vez maior de áreas de pesquisa, constituindo as chamadas Ciências do Esporte, dentre as quais podemos citar disciplinas como a medicina, fisiologia, nutrição, psicologia do esporte, demonstrando uma tendência à interdisciplinaridade, com objetivo de aperfeiçoar cada vez mais o desempenho do atleta e aprimorar a saúde e a qualidade de vida de indivíduos praticantes de atividade física (VIADÉ, 2003; BARA FILHO; MIRANDA, 1998; SAMULSKI, 2002; SOUZA FILHO, 2004; BECKER JÚNIOR, 2000;).

A relação entre esporte e psicologia começou a ser estabelecida e dinamizada no final do século XIX com os primeiros estudos que tentavam identificar as influências dos fatores psicológicos no rendimento de atletas. A partir de estudos desenvolvidos por Colleman Grifth essa relação passou a ser melhor definida.Novos laboratórios surgiram nos Estados Unidos e na Europa aumentando consideravelmente o número de investigações e também a sua qualidade. A partir de 1965, quando Ferrucio Antonelli e Antonio Salvini realizaram o Primeiro Congresso Mundial de Psicologia do Esporte (em Roma), essa disciplina entrou definitivamente no rol das chamadas "Ciências do Esporte". (CORTEZ, DE ROSE JÚNIOR, KNIJNIK, SIMÕES, 2004; WEINBERG; GOULD, 2001).

A Psicologia do Esporte é a disciplina acadêmica que tem como objeto de estudo as diferentes dimensões psicológicas da conduta humana no contexto do esporte e da atividade física. Investiga as causas e os efeitos de ocorrências psíquicas que o ser humano apresenta antes, durante e após o exercício, sendo estes educativos, recreativos, competitivos ou reabilitador. (BECKER JR, 2000).

A teoria do treinamento esportivo é a que rege a maior parte das ciências aplicadas ao esporte. O atleta é o protagonista, o personagem principal, sem o qual o esporte não existiria, e para alcançar o sucesso necessita de preparação e muito treinamento (DOSIL, 2004). Esse treinamento é elaborado com 
antecedência por toda a comissão, para que o trabalho seja continuo e progressivo, tendo como meta o bom desenvolvimento do esportista. Mas, apesar de ser considerado ideal, poucos são os treinamentos que possuem algum embasamento cientifico, seja por questões econômicas e políticas, ou por falta do conhecimento dos profissionais que atuam no meio (SANTOS, 2000).

A preparação física, preparação técnica, preparação tática e preparação psicológica são fatores dos quais os atletas dependem para competir. Devem ser tratados com a mesma importância, e juntos podem oferecer ao atleta ou à equipe melhores condições de alcançar os resultados almejados. Dependendo do momento ou da fase da preparação em relação a uma competição, cada um deles assume grande importância (BARA FILHO; MIRANDA, 1998).

A preparação física é essencial durante todo o processo, desde a aquisição das habilidades fundamentais para desempenhar adequadamente, até a manutenção do que foi adquirido. A preparação técnica ocorre simultaneamente à preparação física, e é composta pela aprendizagem e pelo aperfeiçoamento dos gestos básicos de cada modalidade esportiva e suas variações, progredindo para a preparação tática que é enfatizada em fases mais próximas da competição.

E também nesse período que se destaca o aspecto psicológico, que vem ganhando cada vez mais ênfase no desporto de alto rendimento, para a melhoria de desempenho do atleta. De acordo com Feijó (1998), o preparo psicológico tem sido usado por atletas, treinadores, dirigentes e jornalistas para explicar derrotas e vitórias. No entanto, apesar de sempre utilizado, raramente é bem definido. Devido a essa falta de definição e conhecimento é que para alguns a Psicologia do Esporte se limita a apenas aplicação de algumas técnicas psicológicas para aqueles momentos de crise vividos pelos clubes.

Em uma equipe o posto central é ocupado pelo técnico e este tem a responsabilidade maior de organizar e colocar em prática os planos elaborados para a equipe, é ele que toma as principais decisões. Em um treinamento de alto rendimento, o ideal seria que uma equipe técnica fosse formada por diferentes profissionais. Cada um desses especialistas utilizaria os métodos e técnicas especificas de sua área para desenvolver seu trabalho, com o objetivo de oferecer o suporte ideal para a preparação integral do atleta. Neste contexto, o trabalho do psicólogo do esporte deveria ser considerado de maneira natural, como componente dessa estrutura esportiva. Todos os caminhos a serem percorridos seriam planejados e analisados por essa equipe através de um trabalho visando o ótimo desempenho do esportista. 
Porém, isso nem sempre acontece devido à falta de estrutura das equipes, à falta de informação e, até mesmo de boa vontade dos envolvidos com ela (BARA FILHO; MIRANDA, 1998). A maioria das equipes brasileiras não dispõe de um psicólogo esportivo inserido na comissão técnica, e às vezes este só é lembrado quando o time está em dificuldades, quando alguns se arriscam a dar uma palestra ou dicas para a equipe, sem dar qualquer continuidade do trabalho. De Rose JR. apud Santos 2000, p. 77, critica a falta de consciência da necessidade da preparação psicológica e a falta de espaço no campo prático para as investigações cientificas.

O treinamento desportivo se define como "um processo mediante o qual são produzidas adaptações sucessivas diante de estímulos que levam a uma melhora do rendimento" (DOSIL 2004). Nesse processo de adaptação não só o aspecto físico ocupa um lugar importante, mas também o mental, que se converte no elemento chave, pois a capacidade de lidar com diferentes aspectos psicológicos envolvidos em uma competição é que pode determinar a diferença entre o atleta vencedor e o perdedor ou entre o atleta verdadeiramente talentoso e o comum. Por tudo isso, a relação que existe entre o treinamento e a psicologia é íntima desde o momento em que não se é possível separar a relação corpo-mente. (DOSIL, 2004).

Isto posto, o objetivo do presente estudo foi verificar a importância da psicologia do esporte para treinadores esportivos.

\section{METODOLOGIA}

Amostra

O presente estudo foi realizado com 59 treinadores (idade média 34,98 \pm 10,26 e tempo de prática 9,67 \pm 8,91 ), sendo $88,1 \%$ do sexo masculino e $11,9 \%$ do sexo feminino, de modalidades coletivas $(77,2 \%)$ e individuais $(22,8 \%)$. Todos filiados a sua respectiva federação.

\section{INSTRUMENTO}

Foram utilizados os seguintes instrumentos de investigação para a obtenção dos dados:

-Questionário geral, contendo as questões sobre: gênero, idade, modalidade esportiva, tempo de treinamento. 
Questionário específico, contendo as seguintes questões:

- Qual importância dos aspectos técnico, tático, físico e psicológico no treinamento esportivo (Escala tipo Likert: 0 - Não sei; 1 - Nenhuma; 2 - Pouca; 3 Razoável; 4 - Importante; 5 - Muito importante).

- Possui conhecimento acerca dos aspectos acima citados (Escala tipo Likert: 0 - Não sei; 1 Nenhum; 2 - Pouco; 3 - Razoável; 4 - Elevado; 5 - Muito elevado).

- Já trabalhou com um psicólogo esportivo?

- Qual nível de satisfação com o trabalho do psicólogo esportivo (Escala tipo Likert: 0 - Não sei; 1 - Nenhuma; 2 - Pouca; 3 - Razoável; 4 - Suficiente; 5 Alto).

- Qual importância do psicólogo do esporte na comissão técnica (Escala tipo Likert: 0 - Não sei; 1 - Nenhuma; 2 - Pouca; 3 - Razoável; 4 - Importante; 5 Muito importante)?

Foi perguntado também se os técnicos concordavam ou não com algumas afirmações sobre a presença do psicólogo na equipe técnica:

- O psicólogo possui o conhecimento necessário.

- O psicólogo conhece as características do esporte.

- O psicólogo deve ser o principal responsável para trabalhar os aspectos psicológicos.

- O psicólogo deve atuar auxiliando o treinador.

- O treinador deve ser o principal responsável para trabalhar as variáveis psicológicas.

- O treinador deve possuir os conhecimentos necessários para trabalhar o desenvolvimento dos aspectos psicológicos. 


\section{ANÁLISE DOS DADOS}

Para a análise dos dados foram feitas as seguintes técnicas estatísticas: cálculo da média, desvio padrão e percentuais da amostra para as variáveis do questionário (Thomas e Nelson, 2002).

A presente pesquisa atendeu as determinações da Declaração de Helsinque e a resolução 196/96 do Conselho Nacional de Saúde. O projeto foi previamente aprovado pelo Comitê de Ética e Pesquisa da Universidade Federal de Juiz de Fora. Os participantes da pesquisa assinaram um Termo de Consentimento antes de responderem ao questionário.

\section{RESULTADOS}

Figura 1 - Importância dos aspectos técnico, tático, fisico e psicológico.

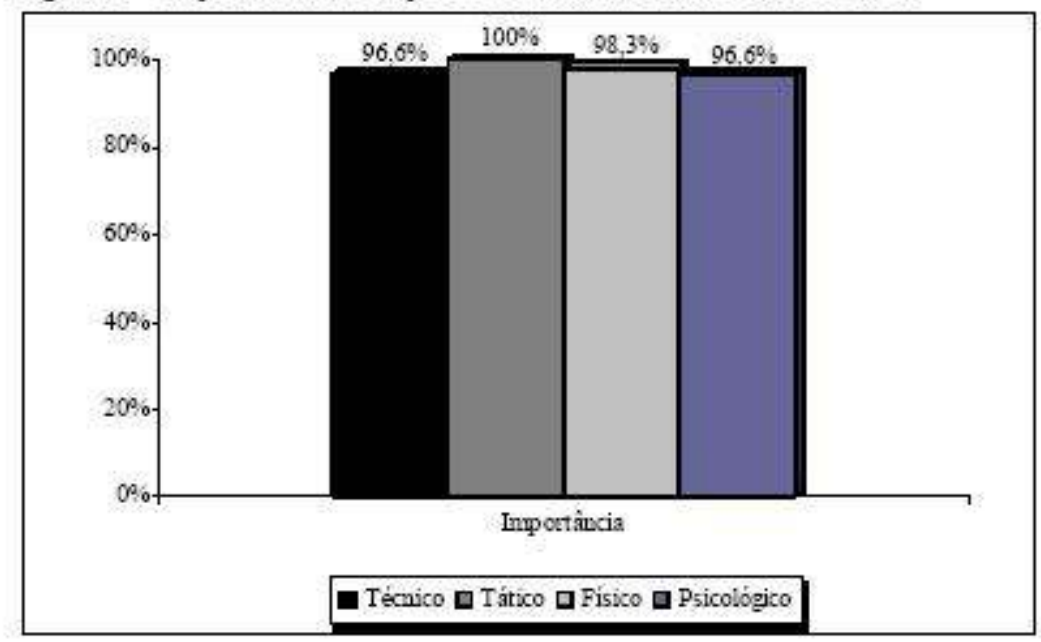

Quando questionados a respeito da importância dos aspectos do treinamento esportivo (Figura 1), verificou-se que a grande maioria dos treinadores considera os quatro fatores (técnico, tático, físico e psicológico) importantes e devem ser trabalhados para que a preparação do atleta seja completa. Observase que os aspectos possuem diferentes níveis de relevância para os técnicos: Técnico: 96,6\%; Tático: 100\%; Físico: 98,3\% e Psicológico: 96,6\%. 
Figura 2 - Conhecimento dos treinadores.

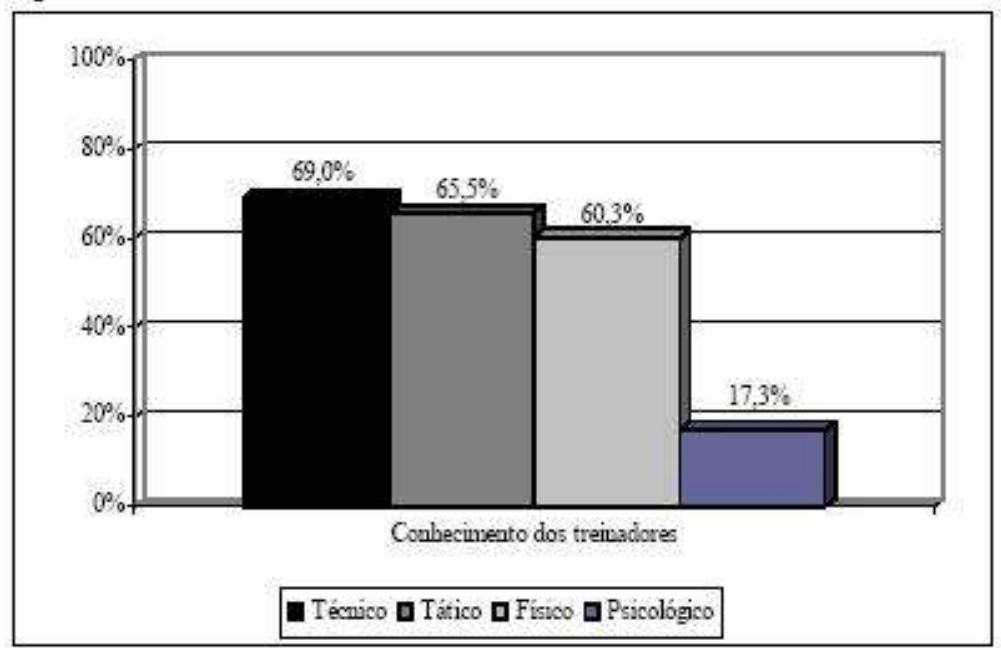

Dos técnicos que responderam ter um conhecimento elevado ou muito elevado a respeito dos aspectos relevantes para o treinamento esportivo (Figura 2) percebe-se um equilíbrio no entendimento das variáveis: técnica (69\%), tática $(65,5 \%)$ e física $(60,3 \%)$. Entretanto, observa-se que o conhecimento sobre o aspecto psicológico ainda é muito restrito, pois apenas $17,3 \%$ dos técnicos relataram ter um cnhecimento elevado ou muito elevado sobre a variável psicológica.

Quando indagados se já haviam trabalhado com um psicólogo esportivo na equipe, a maioria dos técnicos respondeu negativamente $(57,6 \%)$. Entre os treinadores que tiveram essa experiência $(42,4 \%)$, o nível de satisfação variou entre razoável (28\%), suficiente (48\%) e alto (24\%).

Também foram perguntados se concordavam ou não com algumas afirmações a respeito do psicólogo na equipe esportiva (Tabela 1). 


\begin{tabular}{lc}
\hline \multicolumn{1}{c}{ Questão } & Concordo \\
\hline O psicólogo possui o conhecimento necessário. & $76,3 \%$ \\
O psicólogo conhece as características do esporte. & $52,5 \%$ \\
O psicólogo deve ser o principal responsável para trabalhar & $66,1 \%$ \\
os aspectos psicológicos. & \\
O psicólogo deve atuar auxiliando o treinador. & $91,5 \%$ \\
O treinador deve ser o principal responsável para trabalhar & $39,0 \%$ \\
as variáveis psicológicas. & \\
O treinador deve possuir os conhecimentos necessários para & $74,6 \%$ \\
trabalhar o desenvolvimento dos aspectos psicológicos. & \\
\end{tabular}

Tabela 1 - O psicólogo do esporte na equipe.

Analisando a Tabela 1, observou-se que 76,3\% dos treinadores concordam que o psicólogo do esporte tem o conhecimento necessário para realizar seu trabalho e $66,1 \%$ afirmam que ele deve ser o principal responsável para trabalhar os aspectos psicológicos com os atletas, porém apenas 52,5\% acreditam que o psicólogo tem o conhecimento das características do esporte em que trabalha e provavelmente, por isso, para a grande maioria dos técnicos $(91,5 \%)$ o psicólogo do esporte deve atuar apenas auxiliando o treinador.

Quando perguntados se o treinador deve ser o principal responsável para trabalhar as variáveis psicológicas, 39\% dos técnicos concordaram, porém $74,6 \%$ acreditam que o técnico deve possuir os conhecimentos necessários para trabalhar o desenvolvimento dos aspectos psicológicos dos seus atletas (Tabela 1).

Finalmente, 96,6\% dos treinadores responderam que o psicólogo esportivo dentro da comissão técnica, é importante ou muito importante.

\section{CONSIDERAÇÕES FINAIS}

A partir da analise dos resultados podemos concluir que os treinadores consideram os quatro aspectos do treinamento desportivo (técnico, tático, físico, psicológico) importante ou muito importante. 
Porém, o conhecimento acerca do aspecto psicológico ainda é baixo, tendo em vista que apenas 17,3\% dos técnicos relataram ter algum conhecimento a respeito deste conteúdo.

O percentual de treinadores que relataram j á ter trabalhado com um psicólogo esportivo é considerável, quase metade $(42,4 \%)$. Porém são necessárias investigações que verifiquem se estes profissionais eram realmente qualificados para trabalhar como psicólogo do esporte.

Conclui-se também, que apesar de considerarem a presença do psicólogo do esporte na comissão técnica importante $(96,6 \%)$, e acreditarem que este poderia atuar auxiliando o trabalho dos técnicos $(91,5 \%)$, eles ainda têm um receio acerca do conhecimento que os psicólogos possuem sobre a pratica esportiva, tendo em vista que $52,5 \%$ dos treinadores concordaram que o psicólogo conhece as características do esporte.

São necessários futuros estudos que verifiquem quais são os temas específicos da psicologia do esporte que interessam para treinadores e atletas a fim de traçar um paralelo entre as necessidades dos esportistas com a psicologia do esporte, e o que esta estuda tentando aproximar o trabalho do psicólogo do esporte na teoria e a pratica esportiva.

\section{REFERÊNCIAS}

BARA FILHO, M. G.; MIRANDA, R. Aspectos psicológicos do esporte competitivo. Revista Treinamento Desportivo, Curitiba, n. 3, p. 62-72, 1998.

BECKER JUNIOR, B. Manual de psicologia do esporte \& exercício. Porto Alegre: Nova Prova, 2000.

DE ROSE JR, D. História e evolução da psicologia do esporte. Revista Paulista de Educação Física, São Paulo, n. 6, p. 73-78, 1992.

DOSIL, J. Psicología de la activiade fisica y del deporte. Madrid: McGrawHill; 2004.

FEIJÓ, O. G. Psicologia para o esporte: corpo e movimento. 2.ed. Rio de Janeiro: Shape, 1998.

FONSECA A. M. A psicologia do desporto e a batalha da qualidade. Rev. Port. De Ciência do Desporto, v. 1, n. 1, p. 114-123, 2001.

GARCÉS DE LOS FAYOS; E.; VIVES, L. Los límites del entrenamiento psicológico aplicado al deporte. Cuadernos de Psicología del Deporte, Murcia, v.5, n. 1/2, p. 273- 285, 2005.

GARCIA, M. A. S. A. La psicologia del deporte y sus relaciones con otras ciencias del deporte. Revista de Psicología del Deporte, Barcelona, v.11, n. 1, p. 103-113, 1997. 
OLMEDILLA, A; GARCÍA, C.; GARCÉS DE LOS FAYOS, E.Un análisis del papel profesional del deporte desde la percepción del entrenador de fútbol. Revista de Psicologia del Deporte, Barcelona, v. 13, n. 1, p. 95-111, 1998.

OLMEDILlA, A, et al. Formación en psicología para entrenadores de fútbol: una propuesta metodológica. Revista de Psicologia del Deporte, v. 13, n. 2, p. 247-262, 2004.

SAMULSKI, D. Psicologia do esporte. Barueri: Manole, 2002.

SANTOS, S. G.; SHIGUNOV, V. Suporte psicológico ao atleta: uma necessidade "teórica" que precisa ser aplicada. Revista Treinamento Desportivo, Curitiba, v. 5, n. 2, p. 74-83, 2000.

SIMÕES A. C.; et. al. A psicossociologia como área de conhecimento da ciência do esporte. Revista Paulista Educação Física, São Paulo, v.18. p.73-81, ago. 2004.

SOUSA FILHO, P. G. O que é psicologia dos esportes? Revista Brasileira de Ciência e Movimento, v.8, n.4, p. 33-36, 2000.

THOMAS, J. R.; NELSON, J. K. Métodos de pesquisa em atividade física. Porto Alegre: Artmed, 2002.

VIADÉ, A. Psicología del rendimiento deportivo. Barcelona: UOC, 2003.

WEINBERG, R. S.; GOULD, D. Fundamentos da psicologia do esporte e do exercício. Porto Alegre: Artmed, 2001. 
Danilo Reis Coimbra

Universidade Federal de Juiz de Fora

Simone Salvador Gomes

Universidade Federal de Juiz de Fora

Felipe Carvalho

Universidade Estácio de Sá- RJ

\section{Renato Ferreira \\ UNIBH - MG}

\section{Felix Guillen}

Universidad de Las Palmas de Gran Canaria- Espanha

\section{Renato Miranda}

Universidade Federal de Juiz de Fora

\section{Mauricio Bara Filho}

Universidade Federal de Juiz de Fora

\section{Referência do artigo:}

\section{ABNT}

COIMBRA, D. R. et al. Importância da psicologia do esporte para treinadores. Conexões, v. 6, p. 419-429, 2008.

\section{APA}

Coimbra, D. R., Gomes, S. S., Carvalho, F., Ferreira, R., Guillen, F., Miranda, R., \& Filho, M.B. (2008). Importância da psicologia do esporte para treinadores. Conexões, 6, 419-429.

\section{VANCOUVER}

Coimbra DR, Gomes SS, Carvalho F, Ferreira R, Guillen F, Miranda R, Filho MB. Importância da psicologia do esporte para treinadores. Conexões, 2008; 6: 419-429. 